\title{
A PECULIAR UNMIXED DOMAIN
}

\author{
MARKUS BRODMANN AND CHRISTEL ROTTHAUS
}

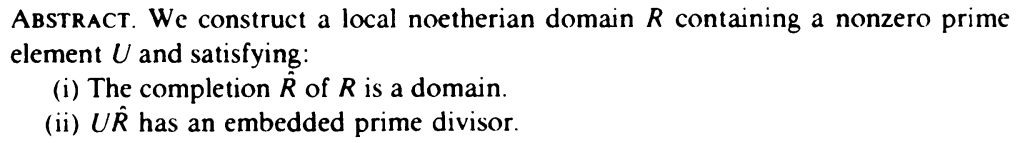

In [7] Nagata asked the following question: Let $(R, \mathrm{~m})$ be a local noetherian domain and let $\mathfrak{p} \in \operatorname{Spec}(R)$. Assume that $R$ is unmixed (which means that $\operatorname{dim}(\hat{R} / \mathfrak{p})=\operatorname{dim}(R)$ for all $\hat{\mathfrak{p}} \in \operatorname{Ass}(\hat{R}))$. Is $R / \mathfrak{p}$ unmixed again?

Clearly, an example as mentioned in the abstract, gives a negative answer to this question.

The method we propose to obtain $R$ follows partly the method used in [6]. Originally we used a construction based on a similar principle presented in [2]. We are grateful to the referee for having pointed out to us that modifying the approach of [6] is more effective in getting the type of ring in question. We also thank him for his helpful comments, in particular the use of (9), which essentially simplified some of our original arguments.

We want to point out the essential difference between the rings constructed in [2] and the ring which will be constructed in this paper. The main result of [2] states:

(1) There is a countable field $K$ (which may be chosen of any characteristic) such that - with a set $X, Y, V_{1}, \ldots, V_{m}$ of indeterminates-the following holds: Let a $\subseteq$ $K\left[V_{1}, \ldots, V_{m}\right]$ be an ideal consisting of polynomials without constant term. Then there is a local domain $(R, \mathrm{~m})$ such that:

(i) $\hat{R}=K \llbracket X, Y, V_{1}, \ldots, V_{m} \rrbracket /(a)$.

(ii) $\overline{\mathrm{a}}:=\left(V_{1}, \ldots, V_{m}\right) \hat{R}$ is prime and satisfies $\overline{\mathrm{q}} \cap R=(0)$.

(iii) If $\mathfrak{p} \in \operatorname{Spec}(R-\{(0)\}), R / \mathfrak{p}$ is essentially of finite type over $K$.

So (1) allows us to construct examples with arbitrarily "bad" generic formal fibers ((i) and (ii)) and with "good" formal fibers at nonzero primes (iii). On the other hand, to deny Nagata's question we need a ring of the contrary shape. Its generic formal fiber has to be better than the formal fiber in some nonzero prime.

We start our construction with a preliminary remark. Let $K$ be a field and let $U, V, W, T$ be indeterminates. Let $\mathfrak{S}_{0} \subseteq K[U, V, W, T]$ be the prime ideal generated by $P_{1}=W^{3}-T^{2}, P_{2}=W U^{2}-V^{2}, P_{3}=W V-U T, P_{4}=W^{2} U-T V$. This is exactly the prime treated as an example in [5]. We know for example that there is a

Received by the editors October 5, 1981 and, in revised form, July 29, 1982.

1980 Mathematics Subject Classification. Primary 13C15, 13H 10.

Key words and phrases. Local domain, completion, embedded prime, unmixedness.

(C)1983 American Mathematical Society $0002-9939 / 82 / 0000-1122 / \$ 02.00$ 
morphism $\mathrm{A}_{K}^{2} \rightarrow \operatorname{Spec}\left(K[U, V, W, T] / \mathfrak{I}_{0}\right)$, which is a (Zariski-) homeomorphism, and an isomorphism apart from the origin. $\operatorname{So} \operatorname{Spec}\left(K[U, V, W, T] / \mathfrak{S}_{0}\right)$ has a unique singularity - namely the origin (from which we moreover know that it is of proper Buchsbaum-type [4]). In particular $\left(K[U, V, W, T] / \mathfrak{S}_{0}\right)_{(U, V . W . T)}$ is a local domain of dimension 2 and of depth 1.

Now, to perform our construction we start according to [6] (with the modification of introducing an additional variable $U$ ). So let $k$ be an infinite-countable field and let $A_{1 n}, A_{2 n}, A_{3 n}(0 \leqslant n \leqslant \infty)$ be indeterminates. Put $K_{m}=$ $k\left(A_{1 j}, A_{2 j}, A_{3 j} / j \leqslant m\right)$ and $K=\cup K_{m}$. Let $X, U, Z_{1}, Z_{2}, Z_{3}$ be additional variables. Put $Q_{0}=K\left[X, Z_{1}, Z_{2}, Z_{3}, U\right], \mathrm{r}_{0}=\left(X, Z_{1}, Z_{2}, Z_{3}, U\right), Q=\left(Q_{0}\right)_{r_{0}}, \mathrm{r}=\mathrm{r}_{0} Q$. Now we find a set $\mathfrak{B}$ of prime elements in $Q_{0}$ with the following properties:

(2)(a) $X \in \mathfrak{B}$.

(b) $p, q \in \mathfrak{B}, p \neq q \Rightarrow p Q \neq q Q$.

(c) If $\mathfrak{p} \in \operatorname{Spec}(Q-\{U Q\})$ is a prime ideal of height one, $\mathfrak{p}$ is generated by an element $p \in \mathfrak{B}$.

(d) If $q \in U \cdot Q$ is a prime element, then $q \notin \mathfrak{B}$.

Now we may choose an enumeration $\mathbf{N} \stackrel{\delta}{\rightarrow} \mathfrak{B}$ of $\mathfrak{B}$ such that (with $\delta(n)=p_{n}$ ):

(3)(a) $p_{1}=X$.

(b) $p_{n} \in K_{n-2}\left[X, Z_{1}, Z_{2}, Z_{3}, U\right](n>1)$.

Next, define the following elements and ideals.

(4)(a) $q_{n}:=\prod_{j=1}^{n} p_{j}(n \in \mathbf{N})$,

(b) $\xi_{i 0}:=Z_{i}, \xi_{i n}=Z_{i}+\sum_{j=1}^{n} A_{i j} q_{j}^{j}(i=1,2,3)$,

(c) $\mathfrak{p}_{n}:=\left(\xi_{1 n}, \xi_{2 n}, \xi_{3 n}, U\right) Q(n \geqslant 0)$.

Observe that $\xi_{1 n}, \xi_{2 n}, \xi_{3 n}, U$ make part of a regular system of parameters for $Q$. So $p_{n}$ is a prime ideal of height 4 .

(5) Proposition. $p_{n} \notin \mathfrak{p}_{n-1}, \forall n \in \mathbf{N}$.

Proof. See [6] (the arguments are not affected by the presence of the additional variable $U$ ).

Now, put $A=K[U, V, W, T]_{(U, V . W . T)}$ and (using the above notation) $\mathfrak{s}=\mathfrak{s}_{0} A$, $\mathfrak{t}=(U, V, W, T) A$. Next we define

(6)(a) $\pi_{i n}:=P_{i}\left(U, \xi_{1 n}, \xi_{2 n}, \xi_{3 n}\right)(i=1, \ldots, 4)$,

(b) $\mathfrak{Q}_{n}:=\left(\pi_{1 n}, \ldots, \pi_{4 n}\right) Q$.

As $U, \xi_{1 n}, \xi_{2 n}, \xi_{3 n}, X$ are a regular system of parameters for $Q$, we have $\hat{Q}=K \llbracket U, \xi_{1 n}, \xi_{2 n}, \xi_{3 n}, X \rrbracket$, where ${ }^{\prime}$ denotes the completion. So there is a canonical isomorphism

$$
\begin{aligned}
\hat{A} \llbracket X \rrbracket=K \llbracket X, U, V, W, T \rrbracket \stackrel{\beta_{n}}{\longrightarrow} \hat{Q} \quad & \text { with } \beta_{n}(X)=X, \beta_{n}(U)=U, \\
& \beta_{n}(V)=\xi_{1 n}, \beta_{n}(W)=\xi_{2 n}, \beta_{n}(T)=\xi_{3 n} .
\end{aligned}
$$

$\beta_{n}$ sends $\mathfrak{B} \hat{A}[X]$ to $\mathfrak{Q}_{n} \hat{Q}$. As the integral closure of $A / \mathfrak{S}$ is a (regular) local domain

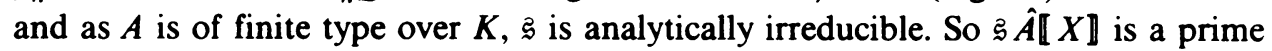
ideal (of height 2). Thus $\mathfrak{Q}_{n} \hat{Q}$, hence in particular $\mathfrak{Q}_{n}$, is a prime ideal of height 2 . 
Let $L$ be the quotient field of $Q$. As $\pi_{i n-1} \in\left(\pi_{i n}, q_{n}^{n}\right) Q$ (use (4) and (6)) in $L$ we obviously have the relation

$$
q_{n-1}^{-(n-1)} \Omega_{n}, \subseteq q_{n} Q\left[q_{n}^{-n} \Sigma_{n}\right] .
$$

In particular it follows $Q\left[q_{n-1}^{-(n-1)} \Sigma_{n} 1\right] \subseteq Q\left[q_{n}^{-n} \Sigma_{n}\right]$. This allows us to define $R^{\prime}=\cup_{n} Q\left[q_{n}^{-n} \Sigma_{n}\right]$ as a subring of $L$.

(9) Lemma. Let $a \in Q$ such that $q_{n}^{n} \in a Q$. Then it holds that $a R^{\prime} \cap Q=\left(a, \Sigma_{n}\right) Q$.

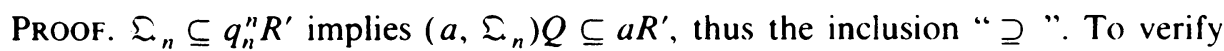
the reverse inclusion it suffices to show that $a Q\left[q_{m}^{-m} \Sigma_{m}\right] \cap Q \subseteq\left(a, \Sigma_{n}\right) Q$ for all $m \gg n$. So let $r=a\left(\alpha_{0}+\cdots+q_{m}^{-m k} \alpha_{k}\right) \in Q \quad\left(\alpha, \in \Sigma_{m}^{\prime}\right)$. It follows that $q_{m}^{m k}\left(r-a \alpha_{0}\right) \in \Sigma_{m}$.

Moreover we have $q_{m} \notin \mathfrak{p}_{m}$. To see this assume the opposite. Then there is a least natural number $s \leqslant m$ with $p_{s} \in \mathfrak{p}_{m}$. By (4) it follows that $\xi_{1(1, \cdots 1)} \in p_{m}(i=1,2,3)$, thus $\mathfrak{p}_{s-1} \subseteq \mathfrak{p}_{m}$. These primes both have height 4 , so they are equal, which induces the contradiction $p_{s} \in \mathfrak{p}_{m}=\mathfrak{p}_{s-1}(\operatorname{see}(5))$.

Now $\Sigma_{m} \subseteq \mathfrak{p}_{m}$ implies $q_{m} \notin \Sigma_{m} . \Sigma_{m}$ being prime, it follows that $\left(r-a \alpha_{0}\right) \in \Sigma_{m}$. thus $r \in\left(a, \Sigma_{m}\right) Q$. By (4) we have $\left(\xi_{1 n}-\xi_{1 m}\right) \in q_{n}^{n} Q$, thus $\left(\pi_{1 n}-\pi_{l m}\right) \in q_{n}^{n} Q \subseteq a Q$. From this we get $\left(a, \Sigma_{m}\right)=\left(a, \Sigma_{n}\right)$, thus $r \in\left(a, \Sigma_{n}\right) Q$.

(10) Corollary. $R^{\prime} / p_{n} R^{\prime}=Q /\left(p_{n}, \Sigma_{n}\right)$.

Proof. (8) (applied for all $m>n$ ) and the observation that $R^{\prime}=\cup_{m \geqslant n} Q\left[q_{n}^{-m} \Sigma_{m}\right]$ show that $R^{\prime}=Q+p_{n} R^{\prime}$. Thus the map $Q \rightarrow R^{\prime} / p_{n} R^{\prime}$ is onto. By (9) its kernel is $\left(p_{n}, \Sigma_{n}\right) Q$.

(11) Corollary. $\mathrm{r} R^{\prime}=\left(X, Z_{1}, Z_{2}, Z_{3}, U\right) R^{\prime}$ is a maximal ideal of $R^{\prime}$ and $R^{\prime} / \mathrm{r} R^{\prime}$ $=K$.

Proof. Apply (10) with $p_{1}=X$, making use of $p_{1} \in \mathrm{r}$.

Next we put $R=R_{\mathrm{r} R^{\prime}}, \mathrm{m}=\mathrm{r} R$ and consider the following elements in $\hat{Q}=$ $K \llbracket X, Z_{1}, Z_{2}, Z_{3}, U \rrbracket:$

$$
\begin{array}{ll}
\text { (a) } \xi_{i}=Z_{i}+\sum_{j=1}^{\infty} A_{i j} q_{j}^{j} & (1 \leqslant i \leqslant 3), \\
\text { (b) } \pi_{j}=P_{j}\left(U, \xi_{1}, \xi_{2}, \xi_{3}\right) & (1 \leqslant j \leqslant 4) .
\end{array}
$$

(13) Proposition. $R$ is a 3-dimensional local domain and $U R$ is a prime ideal.

Proof. First we show that $U R$ is a prime ideal. So let $f, g \in R$ and assume that $f g \in U R$. Write $f g=U h(h \in R)$. We may choose an element $e \in R^{\prime}-\mathrm{r} R^{\prime}$ such that for an appropriate $n \in \mathrm{N}$ it holds that $e f, e g, e^{2} h \in Q\left[q_{n}^{-n} \check{n}_{n}\right]$. As $e$ is a unit in $R$, it suffices to verify that ef or eg belongs to $U R$. Observing the equality $(e f)(e g)=U\left(e^{2} h\right)$ we may replace $f, g$ and $h$ respectively by $e f, e g$ and $e^{2} h$. So we may assume that $f, g$ and $h$ belong to $Q\left[q_{n}^{-n} \Sigma_{n}\right]$. Choosing $\nu \in \mathbf{N}$ appropriately we have the relations $q_{n}^{n \nu} f, q_{n}^{n \nu} g, q_{n}^{n \nu} h \in Q$, which show that $\left(q_{n}^{n \nu}\right)^{2} f g \in U Q$. As $U Q$ is prime in $Q$, we may assume without loss of generality that $q_{n}^{n v} f \in U Q$. Write $q_{n}^{n \nu} f=U r$. Applying (9) with $n \nu$ instead of $n$ and $q_{n}^{n \nu}=a$ we get $U r \in\left(q_{n}^{n \nu}, \Omega_{n \nu}\right) Q$. 
Write $\bar{Q}=Q / \mathfrak{Q}_{n}$ and denote images modulo $\mathfrak{S}_{n}$ by a bar, $\overline{-}$. Now, with an appropriate $s \in Q$, we may write $\bar{U} \bar{r}=\bar{q}_{n}^{n \nu} \bar{s}$. From the proof of (9) we know that $q_{n \nu} \notin \mathfrak{p}_{n \nu}$, thus $q_{n} \notin \mathfrak{p}_{n \nu}$. On the other side all associated primes of $\left(\Sigma_{n}, U\right)$ are contained in $\mathfrak{p}_{n}$. To see this notice that the isomorphism $\beta_{n}: \hat{A} \llbracket X \rrbracket \stackrel{\simeq}{\rightarrow} \hat{Q}$ maps $\mathfrak{B}=(U, V, W, T) \cdot \hat{A} \llbracket X \rrbracket$ to $\mathfrak{p}_{n \nu} \hat{Q}$ and $(\tilde{\mathfrak{s}}, U) \hat{A} \llbracket X \rrbracket$ to $\left(\Sigma_{n \nu}, U\right) \cdot \hat{Q}$. Now, our claim follows as $\operatorname{Ass}(\hat{A} \llbracket X \rrbracket /(\mathfrak{s}, U))$ has only members contained in $\mathfrak{B}$ (which follows as $\mathfrak{i} \subseteq A, U \in A$ ) and by the faithful flatness of $Q \rightarrow \hat{Q}$.

The above statement shows that $q_{n}$ is regular with respect to $Q /\left(\Sigma_{n \nu}, U\right)$. So $\bar{q}_{n}$ is regular with respect to $\bar{Q} /(\bar{U})$. It follows that $\bar{s} \in \bar{U} \bar{Q}$. Writing $\bar{s}=\bar{U} t$ we obtain $\bar{U} \bar{r}=\bar{q}_{n}^{n \nu} \bar{t} \bar{U}$, thus $\bar{r}=\bar{q}_{n}^{n \nu} \bar{t}$. Thus induces $r \in\left(q_{n}^{n \nu}, \Sigma_{n \nu}\right) Q$. By (9) (applied with $\left.a=q_{n}^{n \nu}\right)$ we get $r \in q_{n}^{n \nu} R^{\prime}$. Consequently $q_{n}^{n \nu} f \in q_{n}^{n \nu} U R^{\prime}$, thus $f \in U R^{\prime}$. This shows that $U R$ is prime.

Now we are ready to show that $R$ is noetherian. So let $\mathfrak{p} \in \operatorname{Spec}(R)-\{(0)\}$. We have to show that $\mathfrak{p}$ is finitely generated. Assume first that $\mathfrak{p} \cap Q \neq U Q$. As $R \subseteq L$ we have $\mathfrak{p} \cap Q \neq(0)$. So there is an $n$ with $p_{n} \in \mathfrak{p}$. (10) shows that $R^{\prime} / p_{n} R^{\prime}$ is noetherian. Thus $\mathfrak{p}$ is finitely generated. Assume now that $\mathfrak{p} \cap Q=U Q$. It suffices to show $\mathfrak{p}=U R$. Assume that this is false. It follows that $U Q\left[q_{n}^{-n} \Sigma_{n}\right] \varsubsetneqq \mathfrak{p} \cap$ $Q\left[q_{n}^{-n} \Sigma_{n}\right]$ for some $n$. So we find an element $f \in \mathfrak{p} \cap Q\left[q_{n}^{-n} \Sigma_{n}\right]-U R$. For a suitable $\nu \in \mathbf{N}$ it must hold that $q_{n}^{n \nu} f \in Q \cap \mathfrak{p}=U Q \subseteq U R$. This induces $q_{n}^{n \prime \prime} \in U R$ $\cap Q=U Q$. This contradicts $(2)(\mathrm{d})$.

It remains to show that $R$ is of dimension 3. By (10) we have $R / p_{n} R=Q /\left(p_{n}, \Sigma_{n}\right)$. Now the result is clear as $R$ is a domain and as the right-hand ring is of dimension 2.

(14) Proposition. There is an isomorphism $\alpha$ : $\hat{A} \llbracket X \rrbracket / s \hat{A} \llbracket X \rrbracket \doteq \hat{R}$ which sends $U \bmod (s \hat{A} \llbracket X \rrbracket)$ to $U$.

Proof. As $\xi_{1}, \xi_{2}, \xi_{3}, X, U$ form a regular system of parameters for $Q$ we may write $\hat{Q}=K \llbracket \xi_{1}, \xi_{2}, \xi_{3}, X, U \rrbracket$, these generators being analytically independent over $K$. So there is a canonical isomorphism $\gamma_{0}: \hat{A} \llbracket X \rrbracket=K \llbracket U, V, W, T, X \rrbracket \rightarrow \hat{Q}$, given by $X \rightarrow X, U \rightarrow U, V \rightarrow \xi_{1}, W \rightarrow \xi_{2}, T \rightarrow \xi_{3}$. By the inclusion map $Q \stackrel{f}{\rightarrow} R$ we get a homomorphism $\hat{\varepsilon}: \hat{Q} \rightarrow \hat{R}$, which maps $U$ to $U$.

We claim that $\hat{\varepsilon}$ is surjective. By (11) the maximal ideal $\mathrm{m} \subseteq R$ is generated by $\left(U, Z_{1}, Z_{2}, Z_{3}, X\right)$ and it holds that $R / \mathrm{m}=K$. Thus we may write $\hat{R}=$ $K \llbracket \hat{\varepsilon}(U), \hat{\varepsilon}\left(Z_{1}\right), \ldots, \hat{\varepsilon}\left(Z_{3}\right) \rrbracket$. This shows the surjectivity of $\hat{\varepsilon}$.

So $\gamma:=\hat{\varepsilon} \cdot \gamma_{0}: \hat{A} \llbracket X \rrbracket \rightarrow \hat{R}$ is surjective. We claim that $\gamma(\therefore \hat{A} \llbracket X \rrbracket)=0$. As $\gamma_{0}\left(P_{,}\right)$ $=\pi_{1}(j=1, \ldots, 4)$ we only have to show that $\hat{\varepsilon}\left(\pi_{1}\right)=0$. To see this observe that $\pi_{1}-\pi_{\jmath n}=q_{n+1}^{n+1} \tau_{\jmath n}$ with a suitable $\tau_{, n} \in \hat{Q}$ for all $n>0$.

In $\hat{R}$ we thus obtain the relation

$$
\begin{aligned}
\hat{\varepsilon}\left(\pi_{j}\right) & =\hat{\varepsilon}\left(\pi_{j n}\right)+\hat{\varepsilon}\left(q_{n+1}^{n+1} \tau_{j n}\right)=\hat{\varepsilon}\left(q_{n}^{n} \frac{\pi_{j n}}{q_{n}^{n}}\right)+\hat{\varepsilon}\left(q_{n+1}^{n+1} \tau_{j n}\right) \\
& =q_{n}^{n} \hat{\varepsilon}\left(\frac{\pi_{j n}}{q_{n}^{n}}\right)+q_{n+1}^{n+1} \hat{\varepsilon}\left(\tau_{j n}\right) \subseteq \mathrm{m}^{n} \hat{R}
\end{aligned}
$$

As this holds for all $n$, our claim follows immediately. 
As already remarked above, $\_A \llbracket X \rrbracket$ is a prime of height 2 . By (13) we have $\operatorname{dim}(\hat{R})=3$. By the previous claim and noticing that $\hat{A} \llbracket X \rrbracket$ is catenary of dimension 5 it follows that $\operatorname{Ker}(\gamma)=\lessgtr \hat{A} \llbracket X \rrbracket$. So $\gamma$ induces an isomorphism $\alpha$ with the requested properties.

(15) COROllary. $R$ is a 3-dimensional local noetherian domain with the following properties:

(i) $\hat{R}$ is a domain,

(ii) $U R$ is a prime ideal.

(iii) $\hat{\mathfrak{D}}:=\alpha(U, V, W, T) / \mathfrak{s} \mathbb{A} \llbracket X \rrbracket$ belongs to $\operatorname{Ass}(\hat{R} / U \hat{R})$ as an embedded component.

Proof. (i) Use (14) and the fact that $\dot{s} \mathbb{A} \llbracket X \rrbracket$ is a prime ideal.

(ii) See (13).

(iii) As $(U, V, W, T) A \in \operatorname{Ass}(A /(\mathcal{B}, U) A)$ the claim follows by (14) and observing that ht $(U, V, W, T) \hat{A} \llbracket X \rrbracket / 亏 A \llbracket X \rrbracket=2$.

So we have constructed a ring as announced in the abstract.

\section{REFERENCES}

I. M. Brodmann. Üher die mimimale Dimension der assoziierten Primideale der Kompletion e'mes lohale'n Integritütshereiches, Comment Math. Helv. 50 (1975), 219-232.

2. M. Brodmann and C. Rothaus, Local domains with had sets of formal prime dicisors. J. Algebra 75 (1982), 386-394

3. D. Ferrand and M. Raynaud. Fihres formelles d'un anneau lecal noetherien. Ann. Sci. Ecole Norm. Sup. 3 (1970), 295-311.

4. S. Goto, On the Cohen-Macaularfication of certain Buchshaum rings. Nagova Math. J. 80 (1980). $107-116$.

5. M. Hochster. Criteria for equality of ordinary and simbolic powers of primes. Math. Z. 133 (1973).

6. R. Heitmann. A non-catenary normal local domain. Rocky Mountain J. Math. 12 (1982), 145-148.

7. M. Nagata. On the chain problem of prime ideals. Nagoya Math. J. 10 (1965). 51-64.

8. T. Ogoma. Non-catenary pseudo-geometric normal rings. Japan J. Math. 6 (1980), 147-163.

9. L. J. Ratliff, Jr.. Chain conjectures in ring theory. Lecture Notes in Math., vol. 647. Springer-Verlag. Berlin and New York. 1978.

10. C. Rotthaus. Unicersell japanische Ringe mit nicht offene'm reguläre'm Ort, Nagoya J. Math. 74 (1979). 123-135.

Department of Mathematics. University of Basel. Rheinsprung 21, CH-4000 Basel

Department of Mathematics. University OF MUNSTER, EINSTEINStrassi: 62. D-4400 MUNSTER. WEST (jERMANY (Current address of Christel Rothaus)

Current address (Markus Brodmann): Mathematisches Institut. University of Zürich. Ramistrasse 74. $\mathrm{CH}$-Zürich. Switzerland 